\title{
Efecto de la velocidad de crecimiento intrahospitalaria en la somatometría a los 2 años en niños con peso de nacimiento menor de 1500 gramos Effect of intrahospital growth rate on somatometric measurements at 2 years old in children with a birth weight of less than 1500 grams
}

\author{
Lic. Salvador Piris Borregas ${ }^{a}$, Dra. María J. Torres Valdivieso ${ }^{a}$, \\ Dip. Cristina Martín-Arriscado Arroba ${ }^{b}$,Dra. María T. Moral Pumarega ${ }^{a}$, \\ Dr. José Beceiro Mosquerac y Dra. Carmen R. Pallás Alonso ${ }^{a}$
}

a. Servicio de

Neonatología, Hospital

Universitario 12 de Octubre. Instituto de Investigación Biomédica i+12,

Universidad Complutense de Madrid, Madrid, España.

b. Instituto de Epidemiología del Hospital Universitario 12 de Octubre, Madrid, España.

c. Departamento de Neonatología, Hospital Universitario Príncipe de Asturias, Madrid, España.

Correspondencia: Lic. Salvador Piris Borregas:

salvador.piris@salud. madrid.org

Financiamiento: Ninguno.

Conflicto de intereses: Ninguno que declarar.

\section{RESUMEN}

Objetivo. Determinar la asociación de la velocidad de crecimiento (VC) intrahospitalaria y la fortificación de leche humana con la somatometría y el riesgo de muerte tras el alta o discapacidad grave a los 2 años de edad corregida (EC).

Población y método. Análisis retrospectivo de la VC y evolución posterior. Se incluyeron los recién nacidos del período 1990-2015, con peso al nacer $<1500 \mathrm{~g}$. Se excluyeron neonatos con patología que afectaba al crecimiento. Se estudió una cohorte global y dos subcohortes: los nacidos en 1990-2001 (sin fortificación) y los nacidos en 2002-2015 (fortificada). La VC se calculó según la fórmula de Fenton y se consideró adecuada si resultó $>12 \mathrm{~g} / \mathrm{kg} /$ día.

Resultados. Se reclutaron 1194 pacientes. La VC adecuada se asoció a mayor mediana de peso a los 2 años de EC en la cohorte global (11 $400 \mathrm{~g}$ [10 300-12 500] versus $11000 \mathrm{~g}$ [10 000-12 140], $p=0,02$. La cohorte 2002-2015 alcanzó mayor talla media $(86,42 \pm 4,03 \mathrm{~cm}$ versus $85,56 \pm 4,01$, $p=0,02)$ y mayor porcentaje de prematuros que alcanzaron un crecimiento compensatorio (catch-up) a los 2 años de EC en la cohorte global con VC adecuada $(62,50 \%$ versus $34,69 \%$, $p<0,02$ ). No se encontraron diferencias en el riesgo de muerte tras el alta o discapacidad grave a los 2 años de EC con VC adecuada (OR: 0,79; IC95\%: 0,47-1,12) ni al considerarse el análisis por subcohortes.

Conclusiones. Una VC adecuada se asoció con mejor crecimiento, pero no con menor riesgo de muerte tras el alta o discapacidad grave. La cohorte fortificada alcanzó mayor talla media a los 2 años de EC.

Palabras clave: unidades de cuidado intensivo neonatal, recién nacido prematuro, crecimiento y desarrollo, nutrición del lactante.

http:/ / dx.doi.org/10.5546/ aap.2021.378

Texto completo en inglés:

http:/ / dx.doi.org/10.5546/ aap.2021.eng.378
Cómo citar: Piris Borregas S, Torres Valdivieso MJ, Martín-Arriscado Arroba C, Moral Pumarega MT, et al. Efecto de la velocidad de crecimiento intrahospitalaria en la somatometría a los 2 años en niños con peso de nacimiento menor de 1500 gramos. Arch Argent Pediatr 2021;119(6):378-385.

\section{INTRODUCCIÓN}

A pesar del descenso de las morbilidades mayores en los recién nacidos prematuros, ${ }^{1}$ la restricción del crecimiento posnatal sigue siendo uno de los problemas principales por resolver en las unidades de cuidados neonatales. ${ }^{2,3}$ Hasta la mitad de los recién nacidos de muy bajo peso (RNMBP), pero con peso adecuado para su edad gestacional, son dados de alta con un percentil de peso inferior a percentil 10 (Pc10) para su edad posmenstrual. ${ }^{4}$ Se conocen los efectos que esa escasa ganancia ponderal intrahospitalaria tiene a largo plazo en el riesgo de muerte o de discapacidad grave, y en la somatometría del prematuro en su período lactante. ${ }^{5-7}$ Varias estrategias, como la fortificación de la leche materna y la leche donada, se han incorporado al manejo nutricional para alcanzar una velocidad de crecimiento (VC) adecuada durante la hospitalización de los RNMBP. Se ha mostrado un efecto positivo en el crecimiento durante la hospitalización, pero se desconoce el impacto de estas estrategias más allá del período neonatal. 
La detección precoz de la restricción posnatal del crecimiento es una prioridad. Se precisan herramientas para monitorizar el crecimiento de los prematuros. La VC es considerada un indicador de calidad de las unidades de cuidados neonatales. ${ }^{8}$ Se han propuesto diversas fórmulas matemáticas con poca concordancia en cuanto a su capacidad predictiva de mortalidad tras el alta o de discapacidad grave. ${ }^{9-13}$ Progresivamente, se ha extendido el uso, tanto en clínica como en investigación, de fórmulas como la propuesta por Fenton, ${ }^{14}$ que simplifican el cálculo y usan la VC media en un período de tiempo determinado. Pero no existe suficiente información sobre la capacidad predictiva de estas fórmulas simplificadas.

El objetivo principal de este estudio fue determinar si existe una asociación entre la VC media intrahospitalaria de los RNMBP, calculada por la fórmula de Fenton, y la somatometría a los 2 años de edad corregida (EC). Como objetivos secundarios, se planteó determinar si existe una asociación entre la VC media intrahospitalaria y la probabilidad de muerte tras el alta o de discapacidad grave a los 2 años, así como estudiar la asociación de la introducción de la fortificación rutinaria de la leche humana en la somatometría y en el riesgo de muerte o discapacidad grave.

\section{POBLACIÓN Y MÉTODO}

El estudio se realizó en una unidad de cuidados neonatales de nivel asistencial IIIC, que realiza un seguimiento de los RNMBP hasta los 7 años. Se efectuó un estudio de cohortes retrospectivo considerando el período 19902015. Se han incluido en el estudio los niños con un peso al nacimiento $\leq 1500 \mathrm{~g}$ durante el período 1990-2015. Se excluyeron los niños con malformaciones mayores o enfermedades genéticas con expresión fetal o neonatal que pudieran influir en el crecimiento, y los que no realizaron un seguimiento completo.

Se consideraron dos cohortes: la cohorte 19902001, que no recibió fortificantes, y la cohorte 2002-2015, que recibió fortificación. La estrategia nutricional que se llevó a cabo en el servicio fue la adición de fortificantes a la leche humana (materna o donada) de forma estándar en todos los prematuros menores de $1500 \mathrm{~g}$ una vez logrado un volumen enteral de $80 \mathrm{ml} / \mathrm{kg} /$ día, que suponía un aporte proteico de entre 3,3 y 3,8 g/ kg/día una vez alcanzados aportes enterales de entre 150 y 175 ml / kg/día. Además, a partir de 2011 se inició la fortificación selectiva cuando, además de haberse alcanzado los aportes enterales exclusivos y habiendo recibido la fortificación estándar, presentaron niveles de urea en sangre $\leq 19 \mathrm{mg}$ / dl, lo que implicó un aporte proteico total de entre 3,5 y $4,2 \mathrm{~g} / \mathrm{kg} /$ día. Durante el tiempo de estudio, la mayoría de los niños (> $80 \%)$ recibieron leche de madre propia, al menos durante parte de la hospitalización. Desde el año 2008 prácticamente el $100 \%$ recibió leche materna propia, leche donada o ambas.

\section{Definición de variables}

La variable dependiente del estudio, la VC media intrahospitalaria, fue calculada mediante la fórmula de Fenton:

$\mathrm{VC}$ media $=([$ peso al alta $(\mathrm{g})-$ peso al nacimiento $(\mathrm{g})] /$ peso medio $(\mathrm{kg}))$ / estancia (días); donde el peso medio $=($ peso al alta $[\mathrm{g}]+$ peso al nacimiento $[\mathrm{g}] / 2$ ).

El resultado de la VC media se expresó en $\mathrm{g} / \mathrm{kg} /$ día. De acuerdo con los criterios de calidad publicados en 2014 para las unidades de cuidados neonatales, ${ }^{7}$ se consideró una VC media adecuada cuando fue mayor o igual a $12 \mathrm{~g} / \mathrm{kg} /$ día.

La variable, resultado del estudio, es la derivada de la somatometría a los 2 años de vida (peso, talla y perímetro craneal). Además, se construyó una variable compuesta para atender el seguimiento del niño, que se ha denominado "muerte tras el alta o discapacidad grave", y que se ha creado con la presencia de alguno de los siguientes eventos hasta los 2 años de EC: muerte tras el alta, agudeza visual menor de 10/100 en el mejor ojo, discapacidad auditiva bilateral sin conseguir audición útil con amplificación (en general mayor de $90 \mathrm{dBL}$ ), parálisis cerebral superior o igual a un grado 3 sobre 5 en la escala Gross Motor Function Classification System, ${ }^{15}$ o un índice de Bayley $\mathrm{II}^{16}$ inferior a 55, un índice de Bayley III ${ }^{17}$ inferior a 65, o contar con la impresión clínica de alteración grave en el neurodesarrollo por parte de un neonatólogo experto en seguimiento del recién nacido prematuro. Respecto al catch-up, se lo definió como el alcance del percentil $\geq 10$ para el parámetro somatométrico considerado (peso, talla o perímetro craneal). Todos estos datos fueron obtenidos de la historia clínica de seguimiento.

Respecto a las variables del período intrahospitalario, se consideraron las siguientes: edad gestacional al nacimiento, sexo, cierre quirúrgico del ductus, presencia de neumotórax, días totales de intubación y oxígeno, días totales de hospitalización, displasia broncopulmonar si 
se precisaba oxígeno a las 36 semanas de edad posmenstrual y retinopatía grave si el grado fue superior a 3 o necesitó terapia con láser. También se consideró la lesión cerebral grave, cuando el paciente presentó un grado de hemorragia intraventricular $\geq$ grado 3 , ventriculomegalia grado 3 o lesión parenquimatosa grado $3 .^{18}$

\section{Análisis estadístico}

No se realizó un cálculo formal del tamaño muestral porque se decidió incorporar a todos los pacientes que cumplieran criterios de inclusión en cada una de las cohortes. Las comparaciones entre las cohortes se realizaron mediante las pruebas no paramétricas de U-Mann-WhitneyWilcoxon o prueba $t$ de Student, y la prueba de chi-cuadrado o prueba exacta de Fisher, según la naturaleza de las variables. La evaluación de los factores de la asociación entre la somatometría a los 2 años de EC y la VC media fue realizada mediante una regresión logística estratificada por la EG, representada por el odds ratio y el intervalo de confianza de Wald del $95 \%$. Se ha considerado un nivel de significación del $5 \%$.

\section{Aspectos éticos}

El estudio fue valorado y aprobado por el Comité Ético de Investigación del Hospital Universitario 12 de Octubre. Durante todo el proceso se ha respetado la Ley Oficial de Protección de Datos, así como la Declaración de Helsinki.

\section{RESULTADOS}

Un total de 2084 recién nacidos prematuros con $\leq 1500 \mathrm{~g}$ de peso al nacer fueron ingresados en la unidad de cuidados neonatales durante el período 1990-2015. En la Figura 1 se muestra el diagrama de flujo tras la aplicación de los criterios de exclusión. En la cohorte 1990-2001, la muestra final fue de 478 pacientes y en la cohorte 20022015, de 716 pacientes.

En la Tabla 1 se muestran las características generales de ambas cohortes, así como el análisis comparativo entre ellas. Cabe destacar que en la cohorte 2002-2015 la edad gestacional media fue significativamente menor (27,99 semanas $\pm 2,01$ versus $28,37 \pm 1,98, p<0,01$ ).
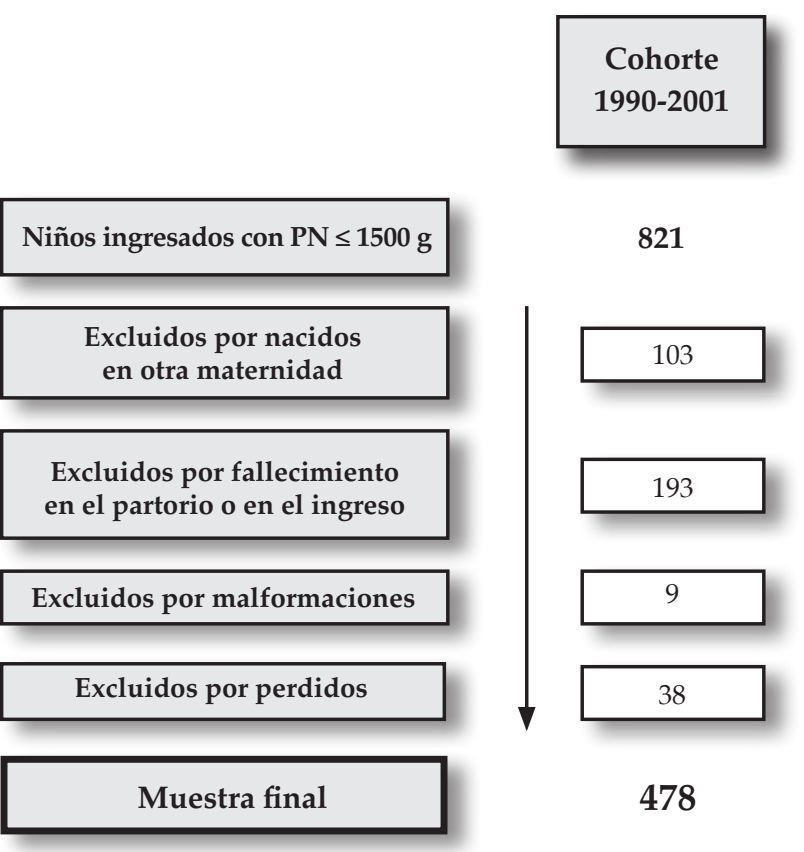

821

Cohorte

2002-2015

1263

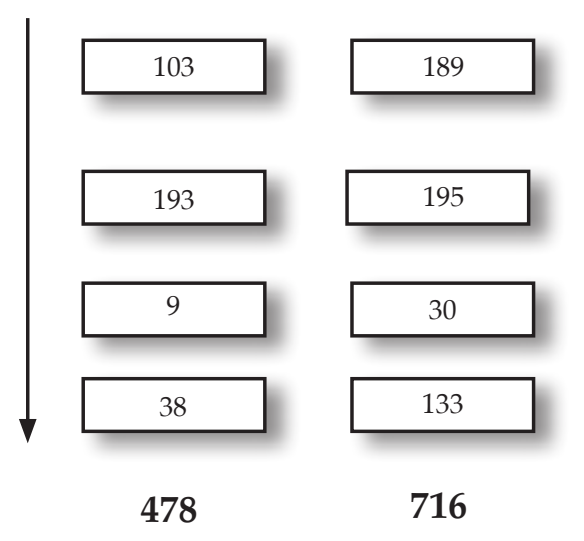

Cohorte global $=1194$

PN: peso de nacimiento. 
En la Tabla 2 se muestran los resultados de la comparación de la somatometría de los niños de la cohorte global y de las dos cohortes temporales a los 2 años de EC considerando si habían alcanzado o no durante el período intrahospitalario una adecuada VC. Cuando los pacientes consiguieron una VC media adecuada durante el período hospitalario, en relación con los que no la habían alcanzado, la mediana de peso a los 2 años de EC fue significativamente mayor tanto en la cohorte global (11400 g [1030012500] versus $11000 \mathrm{~g}$ [10000-12 140], $p=0,02$ ) como en la cohorte fortificada (11420 g [1040012500] versus $11155 \mathrm{~g}$ [10100-12090], $p=0,04)$. Sin embargo, en el caso de la talla, solo en la cohorte 2002-2015, los niños que tuvieron una VC media adecuada tuvieron una mayor talla media a los 2 años de EC $(86,42 \pm 4,03 \mathrm{~cm}$ versus $85,56 \pm 4,01$, $p<0,02$ ).

Se realizó un análisis post hoc del número de recién nacidos prematuros con percentiles de somatometría inferior al Pc10 al nacer que alcanzaron el catch-up a los 2 años de EC en función de la VC media durante el período

TABLA 1. Descripción de la muestra y comparaciones entre las cohortes

\begin{tabular}{lcccc}
\hline Variables & $\begin{array}{c}\text { Cohorte global } \\
\mathbf{N}=\mathbf{1 1 9 4}\end{array}$ & $\begin{array}{c}\text { Cohorte 1990-2001 } \\
\mathbf{N = 4 7 8}\end{array}$ & $\begin{array}{c}\text { Cohorte 2002-2015 } \\
\mathbf{N}=\mathbf{7 1 6}\end{array}$ & $\begin{array}{c}\text { Valor de } \boldsymbol{p} \\
\end{array}$ \\
\hline Sexo femenino (\%) & $576(48,24)$ & $234(48,95)$ & $342(47,77)$ & 0,72 \\
EG en semanas (media/DE) & $28,16(1,99)$ & $28,42(1,95)$ & $27,99(2,00)$ & $<\mathbf{0 , 0 1}$ \\
Peso nacimiento (g) (mediana/RIC) & $1070,00(855,00-1270,00)$ & $1100,00(900,00-1278,00)$ & $1053,00(840,00-1264,00)$ & 0,07 \\
Talla nacimiento (cm) (media/DE) & $36,58(3,21)$ & $36,73(3,12)$ & $36,48(3,26)$ & 0,23 \\
PC nacimiento (cm) (media/DE) & $26,14(2,23)$ & $26,24(2,27)$ & $26,08(2,21)$ & 0,14 \\
ROP grave (\%) (media/DE) & $41(3,43)$ & $22(4,60)$ & $19(2,65)$ & 0,07 \\
O, las 36 semanas (\%) & $202(17,34)$ & $82(18,22)$ & $120(16,78)$ & 0,52 \\
DAP de resolución quirúrgica (\%) & $88(8,72)$ & $25(5,57)$ & $63(11,25)$ & $<0,01$ \\
Lesión cerebral grave (\%) & $137(11,47)$ & $55(11,29)$ & $82(11,34)$ & 0,99 \\
ECN de resolución quirúrgica (\%) & $15(1,58)$ & $4(0,90)$ & $11(2,18)$ & 0,12 \\
Neumotórax (\%) & $36(3,78)$ & $16(3,57)$ & $20(3,96)$ & 0,86 \\
Días de intubación (media/DE) & $1,00(0,00-7,00)$ & $3,00(1,00-9,00)$ & $1,00(0,00-5,00)$ & $<0,01$ \\
Días de oxigenoterapia (media/DE) & $12,50(1,00-49,00)$ & $19,00(3,00-52,00)$ & $10,00(1,00-48,00)$ & $<0,01$ \\
Días de hospitalización (media/DE) & $67,00(51,00-91,00)$ & $70,00(53,00-93,00)$ & $65,50(49,00-89,00)$ & 0,09 \\
\hline
\end{tabular}

EG: edad gestacional; DE: desviación estándar; PC: perímetro craneal; ROP: retinopatía del prematuro; $\mathrm{O}_{2}$ : oxígeno; DAP: ductus arterioso persistente; ECN: enterocolitis necrosante; RIC: rango intercuartílico.

TABla 2. Comparación de las medidas antropométricas a los 2 años de edad corregida considerando una velocidad de crecimiento media intrahospitalaria adecuada o no en la cohorte total y en las diferentes cohortes de estrategias nutricionales. Se muestra también la comparación aplicando un modelo de velocidad de crecimiento ajustada por edad gestacional

\begin{tabular}{|c|c|c|c|c|c|c|c|c|c|c|c|c|}
\hline & \multicolumn{4}{|c|}{$\begin{array}{l}\text { Cohorte total } \\
\qquad N=1194\end{array}$} & \multicolumn{4}{|c|}{$\begin{array}{l}\text { Cohorte } 1990-2002 \\
\qquad N=478\end{array}$} & \multicolumn{4}{|c|}{$\begin{array}{l}\text { Cohorte 2002-2015 } \\
\qquad N=716\end{array}$} \\
\hline & $\begin{array}{c}\text { VC } \\
\text { adecuada } \\
\mathrm{N}=177\end{array}$ & $\begin{array}{c}\text { VC no } \\
\text { adecuada } \\
\mathrm{N}=1017\end{array}$ & $\begin{array}{l}\text { Valor } \\
\text { de } p\end{array}$ & $\begin{array}{l}P \text { ajustada } \\
\text { por EG }\end{array}$ & $\begin{array}{c}\text { a } \quad \mathrm{VC} \\
\text { adecuada } \\
\mathrm{N}=\mathbf{2 9}\end{array}$ & $\begin{array}{c}\text { VC no } \\
\text { adecuada } \\
\mathrm{N}=449\end{array}$ & $\begin{array}{l}\text { Valor } \\
\text { de } p\end{array}$ & $\begin{array}{l}P \text { ajustada } \\
\text { por EG }\end{array}$ & $\begin{array}{l}\text { a } \quad \mathrm{VC} \\
\text { adecuada } \\
\mathrm{N}=\mathbf{1 4 8}\end{array}$ & $\begin{array}{c}\text { VC no } \\
\text { adecuada } \\
\mathrm{N}=568\end{array}$ & $\begin{array}{l}\text { Valor } \\
\text { de } p\end{array}$ & $\begin{array}{l}P \text { ajustada } \\
\text { por EG }\end{array}$ \\
\hline $\begin{array}{l}\text { Peso }(g) \\
\text { (mediana/RIC) }\end{array}$ & $\begin{array}{c}11400 \\
(10300- \\
12500)\end{array}$ & $\begin{array}{c}11000 \\
(10000- \\
12140)\end{array}$ & 0,02 & 0,03 & $\begin{array}{l}11300 \\
(9800- \\
12200)\end{array}$ & $\begin{array}{l}11000 \\
(9960- \\
12200)\end{array}$ & 0,92 & 0,92 & $\begin{array}{c}11420 \\
(10400- \\
12500)\end{array}$ & $\begin{array}{r}11155 \\
(10100- \\
12090)\end{array}$ & 0,04 & 0,049 \\
\hline $\begin{array}{l}\text { Talla }(\mathrm{cm}) \\
(\text { media/DE) }\end{array}$ & $86,16(3,94)$ & $85,26(3,89)$ & 0,02 & $<0,01$ & $84,90(3,21)$ & $84,88(3,69)$ & 0,90 & 0,90 & $86,42(4,03)$ & $85,56(4,01)$ & 0,06 & 0,02 \\
\hline $\begin{array}{l}\text { Perímetro craneal } \\
(\mathrm{cm}) \text { (media/DE) }\end{array}$ & $48,20(1,69)$ & $48,01(1,92)$ & 0,33 & 0,82 & $48,07(1,54)$ & $48,04(1,90)$ & 0,67 & 0,61 & $48,23(1,73)$ & $47,99(1,93)$ & 0,16 & 0,63 \\
\hline $\begin{array}{l}\text { Media EG } \\
\text { (semanas) } \\
\text { (media/DE) }\end{array}$ & $29,01(1,63)$ & $28,02(2,01)$ & $<0,01$ & & $29,69(1,31)$ & $28,33(1,95)$ & $<0,01$ & & $28,87(1,66)$ & $27,77(2,02)$ & & $<0,01$ \\
\hline
\end{tabular}

VC: velocidad de crecimiento; IC: rango intercuartílico; DE: desviación estándar; EG: edad gestacional. 
intrahospitalario. Todos los resultados de este análisis se muestran en la Tabla 3. De ellos, se debe destacar que, en la cohorte global, el porcentaje de recién nacidos con bajo peso para la edad gestacional que alcanzó un percentil 10 de peso a los 2 años de EC fue mayor cuando la VC media intrahospitalaria fue adecuada en comparación con cuando no lo fue $(62,50 \%$ versus $34,69 \%$, $p=0,02)$. Lo mismo se observó cuando se evaluó el porcentaje de recién nacidos que, teniendo un percentil inferior a 10 para el perímetro craneal al nacimiento, alcanzaron el catch-up del perímetro craneal a los 2 años de EC $(79,16 \%$ versus $56,98 \%, p<0,01)$. Cuando se realizó este análisis en las cohortes temporales, los resultados se comportaron de la misma manera para el peso, talla y perímetro craneal en la cohorte 2002-2015, pero no hubo diferencias significativas en la cohorte 1990-2001.

En la Tabla 4 se presenta de forma detallada los niños que han fallecido tras el alta respecto a aquellos que tuvieron una discapacidad

TABla 3. Comparación de la proporción de prematuros con un percentil de peso, talla y perímetro craneal al nacer inferior al percentil 10 que alcanzaron un catch-up a los 2 años de edad corregida en función de que tuvieran una velocidad de crecimiento media intrahospitalaria adecuada o no

\begin{tabular}{|c|c|c|c|c|}
\hline & & VC adecuada & VC no adecuada & Valor de $p$ \\
\hline \multirow[t]{3}{*}{$\begin{array}{l}\text { Pacientes (n) con percentil de peso }<10 \text { al nacimiento } \\
\text { que alcanza el catch-up de peso a los } 2 \text { años de EC (\%) }\end{array}$} & Cohorte global & $\begin{array}{l}30 / 48 \\
(62,5)\end{array}$ & $\begin{array}{l}34 / 98 \\
(34,69)\end{array}$ & 0,02 \\
\hline & Cohorte 1990-2001 & $\begin{array}{c}3 / 7 \\
(42,85)\end{array}$ & $\begin{array}{l}14 / 44 \\
(31,81)\end{array}$ & 0,67 \\
\hline & Cohorte 2002-2015 & $\begin{array}{l}27 / 41 \\
(65,85)\end{array}$ & $\begin{array}{l}20 / 54 \\
(37,03)\end{array}$ & $<0,01$ \\
\hline \multirow[t]{3}{*}{$\begin{array}{l}\text { Pacientes (n) con percentil de talla }<10 \text { al nacimiento } \\
\text { que alcanza el catch-up de talla a los } 2 \text { años de EC (\%) }\end{array}$} & Cohorte global & $\begin{array}{c}34 / 48 \\
(70,83)\end{array}$ & $\begin{array}{c}61 / 98 \\
(62,24)\end{array}$ & 0,35 \\
\hline & Cohorte 1990-2001 & $\begin{array}{c}4 / 7 \\
(57,14)\end{array}$ & $\begin{array}{l}27 / 44 \\
(61,36)\end{array}$ & 1 \\
\hline & Cohorte 2002-2015 & $\begin{array}{l}30 / 41 \\
(73,17)\end{array}$ & $\begin{array}{c}34 / 54 \\
(62,96)\end{array}$ & 0,29 \\
\hline \multirow[t]{3}{*}{$\begin{array}{l}\text { Pacientes (n) con percentil de } \mathrm{PC}<10 \text { al nacimiento } \\
\text { que alcanza el catch-up de PC a los } 2 \text { años de EC (\%) }\end{array}$} & Cohorte global & $\begin{array}{l}38 / 48 \\
(79,16)\end{array}$ & $\begin{array}{l}53 / 93 \\
(56,98)\end{array}$ & $<0,01$ \\
\hline & Cohorte 1990-2001 & $\begin{array}{c}5 / 7 \\
(71,42)\end{array}$ & $\begin{array}{l}23 / 41 \\
(56,09)\end{array}$ & 0,68 \\
\hline & Cohorte 2002-2015 & $\begin{array}{l}33 / 41 \\
(80,48)\end{array}$ & $\begin{array}{c}30 / 52 \\
(57,69)\end{array}$ & 0,01 \\
\hline
\end{tabular}

VC: velocidad de crecimiento; EC: edad corregida; PC: perímetro craneal.

TABla 4. Proporción de los niños fallecidos y de los niños con discapacidad grave a los 2 años de edad corregida en función de la velocidad de crecimiento media intrahospitalaria y de las cohortes nutricionales consideradas

\begin{tabular}{|c|c|c|c|c|c|c|}
\hline & \multicolumn{2}{|c|}{ Cohorte global } & \multicolumn{2}{|c|}{ VC adecuada } & \multicolumn{2}{|c|}{ VC no adecuada } \\
\hline & $\begin{array}{c}\text { Número de } \\
\text { fallecidos/N total } \\
(\%)\end{array}$ & $\begin{array}{c}\text { Número con } \\
\text { discapacidad } \\
\text { grave/N total } \\
(\%)\end{array}$ & $\begin{array}{c}\text { Número de } \\
\text { fallecidos/N total } \\
(\%)\end{array}$ & $\begin{array}{c}\text { Número con } \\
\text { discapacidad } \\
\text { grave/N total } \\
(\%)\end{array}$ & $\begin{array}{c}\text { Número de } \\
\text { fallecidos/N total } \\
(\%)\end{array}$ & $\begin{array}{c}\text { Número con } \\
\text { discapacidad } \\
\text { grave/ } \mathbf{N} \text { total } \\
(\%)\end{array}$ \\
\hline Cohorte global & $\begin{array}{c}111 / 1194 \\
(9,29)\end{array}$ & $\begin{array}{c}76 / 1194 \\
(6,3)\end{array}$ & $\begin{array}{c}16 / 177 \\
(9,03)\end{array}$ & $\begin{array}{l}4 / 177 \\
(2,25)\end{array}$ & $\begin{array}{c}95 / 1017 \\
(9,34)\end{array}$ & $\begin{array}{c}72 / 1017 \\
(7,07)\end{array}$ \\
\hline Cohorte 1990-2002 & $\begin{array}{c}43 / 478 \\
(9)\end{array}$ & $\begin{array}{c}32 / 478 \\
(6,69)\end{array}$ & $\begin{array}{c}0 / 29 \\
(0)\end{array}$ & $\begin{array}{c}3 / 29 \\
(10,34)\end{array}$ & $\begin{array}{c}43 / 449 \\
(9,57)\end{array}$ & $\begin{array}{c}29 / 449 \\
(6,45)\end{array}$ \\
\hline Cohorte 2002-2015 & $\begin{array}{c}68 / 716 \\
(9,49)\end{array}$ & $\begin{array}{c}44 / 716 \\
(6,14)\end{array}$ & $\begin{array}{l}16 / 148 \\
(10,81)\end{array}$ & $\begin{array}{l}1 / 148 \\
(0,67)\end{array}$ & $\begin{array}{c}52 / 568 \\
(9,15)\end{array}$ & $\begin{array}{c}43 / 568 \\
(7,57)\end{array}$ \\
\hline
\end{tabular}

VC: velocidad de crecimiento. 
grave, pero que no llegaron a fallecer. Estos datos se muestran en función de la variable independiente VC y estratificada por las subcohortes nutricionales valoradas en el trabajo. Además, en la Tabla 5, se exponen los resultados de la comparación del riesgo de muerte tras el alta o discapacidad grave a los 2 años de EC considerando la VC media intrahospitalaria. No se encontraron diferencias significativas en la cohorte global ni en las cohortes temporales.

\section{DISCUSIÓN}

En este estudio se muestra cómo, durante las últimas décadas, la población de RNMBP que tuvo una adecuada VC media intrahospitalaria, calculada mediante la fórmula de Fenton, alcanzó mayor peso a los 2 años de EC. En la cohorte donde se introdujo la fortificación de la leche materna, los niños con una VC media adecuada intrahospitalaria también alcanzaron mayor talla a los 2 años de EC; esta cohorte tenía una edad gestacional media significativamente menor. Podría explicarse esta menor edad gestacional media en la cohorte 2002-2015 como un aumento de la complejidad de los pacientes por una actitud cada vez más activa por parte del servicio en trabajar con niños prematuros más pequeños. También, un mayor número de niños con un percentil de peso y perímetro craneal al nacimiento inferior al p10 recuperaron percentiles normales a los 2 años de EC cuando la VC media intrahospitalaria fue adecuada, tanto en la cohorte global como en la cohorte fortificada.

Tan solo se ha encontrado un estudio que ha explorado el impacto de la VC intrahospitalaria en las medidas antropométricas de la infancia temprana. Dicho estudio fue realizado por
Ehrenkranz y cols., ${ }^{2}$ e incluyeron 495 recién prematuros entre $501 \mathrm{~g}$ y $1000 \mathrm{~g}$ de peso. Objetivaron que el porcentaje de niños con percentiles inferiores al 10 para el peso y para la talla a los 18 meses de EC era significativamente menor a medida que aumentaba la VC durante el período neonatal. Estos resultados están en línea con los obtenidos en nuestro estudio.

En relación al impacto de la fortificación proteica de la leche humana en las medidas antropométricas de la infancia temprana, existen estudios que demuestran que esta estrategia nutricional permite mayor ganancia intrahospitalaria ponderal, ${ }^{19}$ de talla ${ }^{20}$ y de perímetro cefálico ${ }^{21}$ hasta las 40 semanas de edad posmenstrual. Nuestros resultados muestran que la cohorte fortificada alcanzó mayor talla a los 2 años de EC, así como un mayor porcentaje de prematuros que alcanzaron el catch-up de perímetro craneal. Algunos estudios comparan diferentes grados de fortificación proteica a largo plazo. Dogra y cols. ${ }^{21}$ no observaron una mejoría significativa de las medidas antropométricas a los 12-18 meses de edad al comparar una fortificación de $1 \mathrm{~g}$ de proteínas $/ 100 \mathrm{ml}$ con otra de 0,4 g/100 ml. Sin embargo, Mariani y cols., ${ }^{22}$ compararon dos regímenes de fortificación proteica de dos unidades neonatales; una con una fortificación proteica de $3,5 \mathrm{~g} / \mathrm{kg} /$ día versus otra de $4,8 \mathrm{~g} / \mathrm{kg} /$ día en una población de neonatos con peso $\leq 1500 \mathrm{~g}$, y encontraron mayor perímetro craneal y mayor talla a los 12 y a los 24 meses en la cohorte fortificada. Por otro lado, hay estudios que apoyan que estrategias como la fortificación han permitido conciliar dos retos actuales de las unidades de cuidados neonatales: aumentar la VC de los niños prematuros sin un detrimento de las

TABla 5. Comparación del riesgo de muerte tras el alta o discapacidad grave a los 2 años de edad corregida considerando una velocidad de crecimiento media intrahospitalaria en la cohorte total y en las cohortes nutricionales. Se muestra también la comparación ajustando la velocidad de crecimiento por edad gestacional

\begin{tabular}{|c|c|c|c|c|}
\hline & VC adecuada & VC no adecuada & & \\
\hline & $\begin{array}{c}\text { Número de fallecidos o } \\
\text { con discapacidad grave / N } \\
\text { con VC adecuada } \\
(\%)\end{array}$ & $\begin{array}{c}\text { Número de fallecidos o } \\
\text { con discapacidad grave/N } \\
\text { con VC no adecuada } \\
(\%)\end{array}$ & OR (IC95 \%) & $\begin{array}{c}\text { OR (IC95 \%) } \\
\text { ajustado por EG }\end{array}$ \\
\hline Cohorte global & $\begin{array}{l}20 / 177 \\
(11,29)\end{array}$ & $\begin{array}{c}167 / 1017 \\
(16,42)\end{array}$ & $0,65(0,38-1,04)$ & $0,79(0,47-1,12)$ \\
\hline Cohorte 1990-2001 & $\begin{array}{c}3 / 29 \\
(10,34)\end{array}$ & $\begin{array}{r}72 / 449 \\
(16,03)\end{array}$ & $0,60(0,14-1,77)$ & $0,84(0,19-2,57)$ \\
\hline Cohorte 2002-2015 & $\begin{array}{l}17 / 148 \\
(11,48)\end{array}$ & $\begin{array}{l}95 / 568 \\
(16,72)\end{array}$ & $0,65(0,36-1,09)$ & $0,80(0,44-1,39)$ \\
\hline
\end{tabular}

VC: velocidad de crecimiento; EG: edad gestacional; OR: odds ratio; IC95\%: intervalo de confianza del $95 \%$. 
tasas de lactancia materna..$^{23}$

Existe bastante evidencia que avala la asociación entre una adecuada VC y un menor riesgo de muerte tras el alta o de discapacidad grave. Numerosos estudios y revisiones sistemáticas muestran una asociación positiva entre una adecuada ganancia ponderal y mejores resultados cognitivos en rangos de edad de entre los 12 meses y los 19 años. ${ }^{2,24-27}$ En nuestro estudio, no se encontró una asociación significativa entre una adecuada VC media intrahospitalaria y un menor riesgo de muerte tras el alta o discapacidad grave a los 2 años de EC. Es posible que la baja frecuencia del evento muerte tras el alta y discapacidad grave no permita encontrar la significación estadística. Por otro lado, existen otros aspectos clínicos del período intrahospitalario tales como la sepsis, la infección nosocomial o el propio retraso del crecimiento intrauterino, así como factores socioeconómicos en la evolución entre el alta hospitalaria y los 2 años de vida que podrían influir en dicha asociación y que no han sido motivo de análisis de este trabajo. ${ }^{28,29}$

El presente estudio es el primero en explorar la relación entre la VC media intrahospitalaria calculada con fórmulas simplificadas y la somatometría más allá del período neonatal. Como limitaciones, debe considerarse que la escasa aparición del evento muerte tras el alta o discapacidad grave en esta muestra, al quedar distribuido por grupos, precisaría un mayor tamaño muestral para estudiar su relación con la VC. Pueden existir otras modificaciones en la práctica clínica relacionadas o no con la asociación entre la VC y la somatometría y el riesgo de muerte tras el alta o discapacidad grave a los 2 años de EC, y que podrían influir en los resultados, por ejemplo, la apertura de un banco de leche humana donada y pasteurizada en el año 2008.

A tenor de los resultados aquí presentados, se puede concluir que la VC media intrahospitalaria mejoró la somatometría a los 2 años de edad. Además, en la población estudiada, la fortificación de leche humana se relacionó con mayor talla y con un porcentaje significativamente mayor de RNMBP que alcanzaron el catch-up de su perímetro craneal en la infancia temprana.

\section{REFERENCIAS}

1. Richter L, Ting J, Muraca G, Boutin A, et al. Temporal Trends in Preterm Birth, Neonatal Mortality, and Neonatal Morbidity Following Spontaneous and Clinician-Initiated Delivery in Canada, 2009-2016. J Obstet Gynaecol Can. 2019;
41(12):1742-51.

2. Ehrenkranz RA, Dusick AM, Vohr BR, Wright LL, et al. Growth in the neonatal intensive care unit influences neurodevelopmental and growth outcomes of extremely low birth weight infants. Pediatrics. 2006; 117(4):1253-61.

3. Cooke RJ, Ainsworth SB, Fenton AC. Postnatal growth retardation: a universal problem in preterm infants. Arch Dis Child Fetal Neonatal Ed. 2004; 89(5):428-30.

4. Horbar J, Ehrenkranz R, Badger G, Edwards E, etal. Weight growth velocity and postnatal growth failure in infants 501 to 1500 grams: 2000-2013. Pediatrics. 2015; 136(1):e84-92.

5. Shah PS, Wong KY, Merko S, Bishara R, et al. Postnatal growth failure in preterm infants: ascertainment and relation to long-term outcome. J Perinat Med.2006;34(6):4849.

6. Franz AR, Pohlandt F, Bode H, Mihatsch W, et al. Intrauterine, early neonatal, and postdischarge growth and neurodevelopmental outcome at 5.4 years in extremely preterminfants after intensive neonatal nutritional support. Pediatrics. 2009; 123(1):e101-9.

7. Sammallahti S, Pyhälä R, Lahti M, Lahti J, et al. Infant growth after preterm birth and neurocognitive abilities in young adulthood. J Pediatr. 2014; 165(6):1109-15.e3.

8. Profit M, Kowalkowski J, Zupancic JA, Pietz K, et al. Babymonitor: a composite indicator of NICU quality. Pediatrics. 2014; 134(1):74-82.

9. Fenton T, Chan H, Madhu A, Griffin I, et al. Preterm infant growth velocity calculations: a systematic review. Pediatrics. 2017; 139(3):e20162045.

10. Fenton T, Anderson D, Groh-Wargo S, Hoyos A, et al. An Attempt to Standardize the Calculation of Growth Velocity of Preterm Infants-Evaluation of Practical BedsideMethods. J Pediatr. 2018; 196:77-83.

11. Briend A, Maire B, Fontaine O, Garenne M. Mid-upper arm circumference and weight-for-height to identify high-risk malnourisher under-five children. Matern Child Nutr. 2012; 8(1):130-3.

12. O'Neill SM, Fitzgerald A, Briend A, Van den Broeck J. Child mortality as predicted by nutritional status and recent weight velocity in children under two in rural Africa. J Nutr. 2012; 142(3):520-5.

13. Chen LC, Chowdhury A, Huffman SL. Anthropometric assessment of energy-protein malnutrition and subsequent risk of mortality among preschool aged children. Am JClin Nutr. 1980; 33(8):1836-45.

14. Fenton T, Nasser R, Eliasziw M, Kim J, et al. Validating the weight gain of preterm infants between the reference growth curve of the fetus and the term infants. BMC Pediatr. 2013; 13:92.

15. Park E. Stability of the gross motor function classification system in children with cerebral palsy for two years. BMC Neurol. 2020; 20(1):172.

16. Kilbride HW, Aylward GP, Doyle LW, Singer LT, Lantos J. Prognostic neurodevelopmental testing of preterm infants: do we need to change the paradigm? J Perinatol. 2017; 37(5):475-9.

17. Spencer-Smith M, Spittle A, Lee K, Doyle L, Anderson P. Bayley-III Cognitive and Language Scales in Preterm Children. Pediatrics. 2015; 135(5):e1258-65.

18. Maller V, Cohen H. Neonatal Head Ultrasound: A Review and Update-Part 1: Techniques and Evaluation of the Premature Neonate. Ultrasound Q. 2019; 35(3):202-11.

19. Moya F, Sisk PM, Walsh K, Berseth CL. A new liquid human milk fortifier and linear growth in preterm infants. Pediatrics. 2012; 130(4):e928-35. 
20. OlsenIE, Harris CL, Lawson ML, Berseth CL. Higher protein intake improves length, not weight, z scores in preterm infants. J Pediatr Gastroenterol Nutr. 2014; 58(4):409-16.

21. Dogra S, Thakur A, Garg P, Kler N. Effect of differential enteral protein on growth and neurodevelopment in infants < $1500 \mathrm{~g}$ : a randomized controlled trial. J Pediatr Gastroenterol Nutr. 2017; 64(5):e126-32.

22. Mariani E, Biasini A, Marvulli L, Maritini S, et al. Strategies of increased protein intake in ELBW infants fed by human milk lead to long term benefits. Front Public Health. 2018; 6:272.

23. Piris Borregas S, López Maestro M, Torres Valdivieso MJ, Martínez Ávila JC, et al. Improving nutritional practices in premature infants can increase their growth velocity and the breastfeeding rates. Acta Paediatr. 2017; 106(5):768-72.

24. Belfort MB, Rifas-Shiman SL, Sullivan T, Collins CT, et al. Infant growth before and after term: effects on neurodevelopment in preterm infants. Pediatrics. 2011; 128(4):899-906.
25. Latal-Hajnal B, von Siebenthal K, Kovari H, Bucher HU, Largo RH. Postnatal growth in VLBW infants: significant association with neurodevelopmental outcome. J Pediatr. 2003; 143(2):163-70.

26. Claas MJ, de Vries LS, Koopman C, Uniken Venema MM, et al. Postnatal growth of preterm born children $</=750$ g at birth. Early Hum Dev. 2011; 87(7):495-507.

27. Ong K, Kennedy K, Castañeda-Gutiérrez E, Forsyth $\mathrm{S}$, et al. Postnatal growth in preterm infants and later health outcomes: a systematic review. Acta Pædiatr. 2015; 104(10):974-86.

28. Krebs N, Lozoff B, Georgieff M. Neurodevelopment: The Impact of Nutrition and Inflammation During Infancy in Low-Resource Settings Pediatrics. 2017; 139(Suppl 1):S508.

29. Zonneberg I, van DijK-Lokkart E, van den Dunjen F, Vermeulen F, van Weissenbruch M. Neurodevelopmental outcome at 2 years of age in preterm infants with late-onset sepsis. Eur J Pediatr. 2019; 178(5):673-80. 